\title{
Free Oscillations of a Toroidal Viscoelastic Shell with a Flowing Liquid
}

\author{
Safarov Ismail Ibrahimovich ${ }^{1}$, Teshaev Muhsin Khudoyberdiyevich ${ }^{2}$, \\ Akhmedov Maqsud Sharipovich ${ }^{2}$ \\ ${ }^{1}$ Department of Mathematics, Tashkent Chemcal - Technological Institute, Tashkent, Republic of Uzbekistan \\ ${ }^{2}$ Department of Mathematics, Bukhara Engineering - Technological Institute, Bukhara, Republic of Uzbekistan
}

Email address:

safarov54@mail.ru (S. I. Ibrahimovich), Muhsin_5@mail.ru (T. M. Khudoyberdiyevich), maqsud.axmedov.1985@mail.ru (A. M. Sharipovich)

\section{To cite this article:}

Safarov Ismail Ibrahimovich, Teshaev Muhsin Khudoyberdiyevich, Akhmedov Maqsud Sharipovich. Free Oscillations of a Toroidal Viscoelastic Shell with a Flowing Liquid. American Journal of Mechanics and Applications. Vol. 6, No. 2, 2018, pp. 37-49. doi: 10.11648/j.ajma.20180602.11

Received: March 20, 2018; Accepted: March 30, 2018; Published: May 7, 2018

\begin{abstract}
On the basis of the method of orthogonal sweep and the Mueller method, the solution of the problem of intrinsic oscillation of a Toroidal shell with a flowing liquid is discussed. The problem of determining the frequencies and forms of intrinsic bending vibrations in the plane of curvature of curvilinear sections of thin-walled Toroidal shells of large diameter with a flowing liquid, with different conditions for fixing the end sections is solved. The behavior of complex Eigen frequencies as a function of the curvature of the shell axis is studied.
\end{abstract}

Keywords: Toroidal Shell, Liquid, Sweep, Mueller Method, Natural Frequency, Oscillation

\section{Introduction}

Elements of structures in the form of a Toroidal shell are widely used in various areas of modern technology. In particular: in the construction of pipelines, power engineering, in the rocket and space industry [1-5], etc. To study the strength and load-bearing capacity of shell structures, it is first of all necessary to determine their stress-strain state, which leads to the need to develop effective methods for solving boundary value problems in shell theory [6-8]. For the first time, the problem of bending vibrations of a straight pipe with a flowing liquid was posed and solved in [9]. Using the equations of small oscillations of the beam, the authors [9] made errors when taking into account the inertial forces of the fluid flow and obtained the wrong result. The error was already corrected in [10], and the solution obtained by him on the beam theory with the help of the Bubnov-Galerkin method of the circular frequency of a hinge fixed at the ends of a pipeline with a steady flow of liquid still has practical application:

$$
\omega_{n}^{2}=\frac{\pi^{4} n^{4}}{L^{4}} \frac{E I}{\left(p A+p_{0} A_{0}\right)}\left(1-\frac{U^{2} p_{0} A_{0} L^{2}}{\pi^{2} n^{2} E I}\right)
$$

where $p, p_{0}$ - the density of the pipe and liquid material, respectively, $A, A_{0}$ - cross-sectional area of pipe and liquid walls, $E I$ - bending stiffness of pipe, $L$ - length of pipeline, $U$ - speed of a flowing liquid. As can be seen from the last formula, the increase in speed $U$ reduces frequency $\omega$. When the speed reaches a certain critical value $U_{k p}$ the oscillation frequency vanishes, and the pipeline loses stability. The value of the critical velocity can be obtained from the last equality:

$$
U_{k p}=\frac{\pi}{L} \sqrt{\frac{E I}{p_{0} A_{0}}}
$$

In [11], this problem was solved by an analytical method, the result obtained was confirmed in [12]. Further studies in this field $[13,14]$ have evolved in the direction of taking into account additional factors affecting the oscillations of the pipelines, and also in the direction of refinement of the solution. The solution of the geometrically nonlinear problem taking into account the influence of internal hydrostatic pressure is considered [15]. In this paper, we solve the problem of the actual vibration of viscoelastic Toroidal shells 
with a flowing fluid, based on orthogonal sweep methods, the Mueller and Gauss method.

\section{Equations of Motion of the Toroidal Shell}

The equation of motion of bending oscillations of the toroidal shell (Figure1) is derived on the basis of the general relations of the geometrically nonlinear theory of shells of the average bending described in [16]. This theory considers such a bending of shells, in which the maximum deflection is of the same order of magnitude as the wall thickness, or even exceeds it, but small compared with other linear dimensions of the shell.

In accordance with this theory, the equations of equilibrium of the moment's forces for the element of the toroidal shell, which is in the deformed state, have the form:

$$
\begin{aligned}
& \frac{\partial}{\partial \beta}\left(A_{2} T_{1}\right)+\frac{\partial}{\partial \theta}\left(A_{1} S_{2}\right)+S_{1} \frac{\partial A_{1}}{\partial \theta}-T_{2} \frac{\partial A_{2}}{\partial \beta}+A_{1} A_{2}\left(\frac{Q_{1}}{R_{1}^{*}}+\tau Q_{2}+X_{1}\right)=0, \\
& \frac{\partial}{\partial \beta}\left(A_{2} Q_{1}\right)+\frac{\partial}{\partial \theta}\left(A_{1} Q_{2}\right)-A_{1} A_{2}\left(\frac{T_{1}}{R_{1}^{*}}+\frac{T_{2}}{R_{2}^{*}}+S_{1} \tau+S_{2} \tau-X_{3}\right)=0, \\
& \frac{\partial}{\partial \beta}\left(A_{2} M_{1}\right)+\frac{\partial}{\partial \theta}\left(A_{1} H_{2}\right)-H_{1} \frac{\partial A_{1}}{\partial \theta}-M_{2} \frac{\partial A_{2}}{\partial \beta}-A_{1} A_{2} Q_{1}=0, \\
& \frac{\partial}{\partial \theta}\left(A_{1} M_{2}\right)+\frac{\partial}{\partial \beta}\left(A_{2} H_{1}\right)+H_{2} \frac{\partial A_{2}}{\partial \beta}-M_{1} \frac{\partial A_{1}}{\partial \theta}-A_{1} A_{2} Q_{2}=0,
\end{aligned}
$$

where $X_{1}, X_{2}, X_{3}$ - components of external force vectors and indices 1 and 2 refer to toroidal coordinate's $\beta$ and $\theta$ respectively.

The first three equations (1) are the equations of equilibrium of forces, the last two are the equations of equilibrium of moments.

Differential equilibrium equations for the shell element (1) are nonlinear, since they contain products of effort and deformation. In addition, they are obtained for a shell in a deformed state. Therefore, these equations include radii of curvature $R_{1}^{*}$ and $R_{2}^{*}$ deformed middle surface of the shell. Their connection with the curvature of the initial state is expressed in accordance with [17] by the following relations:

$$
\frac{1}{R_{1}^{*}}=\frac{1}{R}\left(\cos \theta-\frac{r}{R} \frac{\partial^{2} w}{\partial \beta^{2}}\right), \frac{1}{R_{2}^{*}}=\frac{1}{r}\left(1-\frac{\partial \vartheta}{\partial \theta}\right) .
$$

Change in curvature of the midline of the cross section of the shell $\chi_{2}$ and torsion $\theta$ are expressed in terms of the angle of rotation $\vartheta$ the following relations:

$$
\chi_{2}=-\frac{1}{r} \frac{\partial \vartheta}{\partial \theta}, \theta=-\frac{1}{R} \frac{\partial \vartheta}{\partial \beta}
$$

In accordance with the assumptions (1) - (3) of the semifree shell theory in the first three equilibrium equations (1), we neglect the transverse force $Q_{1}$, and in the last two $H$ the torque. Then (1) of parameters (2) and (3) we obtain a system of equations of motion of the shell in the effort:

$$
\begin{aligned}
& \frac{r}{R} \frac{\partial T_{1}}{\partial \beta}+\frac{\partial S}{\partial \theta}+r \tau Q_{2}+r X_{1}^{*}=0 \\
& \frac{r}{R} \frac{\partial S}{\partial \beta}+\frac{\partial T_{2}}{\partial \theta}+\frac{r}{R} T_{1} \sin \theta+\frac{r}{R_{2}^{*}} Q_{2}+r X_{2}^{*}=0 \\
& \frac{r}{R_{1}^{*}} T_{1}+\frac{r}{R_{2}^{*}} T_{2}+2 r \tau S-\frac{\partial Q_{2}}{\partial \theta}-r X_{3}^{*}=0 \\
& \frac{r}{R} \frac{\partial M_{1}}{\partial \beta}+\frac{\partial H}{\partial \theta}-r Q_{1}=0 \\
& \frac{\partial M_{2}}{\partial \theta}-r Q_{2}=0
\end{aligned}
$$

where $X_{1}, X_{2}, X_{3}$ - components of inertia forces in coordinates $\beta, \theta$ and along the normal to the middle surface, respectively. Eliminating all the forces and moments from equations (4) except $T_{1}$ and $M_{2}$, we arrive at a single equation of motion in the effort:

$$
\begin{aligned}
& \frac{r^{2}}{R^{2}} \frac{\partial^{2} T_{1}}{\partial \beta^{2}}+\frac{r}{R} \frac{\partial}{\partial \beta}\left(\tau \frac{\partial M_{2}}{\partial \theta}\right)+\frac{\partial^{2}}{\partial \theta^{2}}\left(\frac{R_{2}^{*}}{R_{1}^{*}} T_{1}\right)-\frac{1}{r^{2}} \frac{\partial^{2}}{\partial \theta^{2}}\left(R_{2}^{*} \frac{\partial^{2} M_{2}}{\partial \theta_{2}}\right)- \\
& -\frac{\partial}{\partial \theta}\left(\frac{r}{R} T_{1} \sin \theta\right)-\frac{\partial}{\partial \theta}\left(\frac{1}{R_{2}^{*}} \frac{\partial M_{2}}{\partial \theta}\right)+\frac{r^{2}}{R} \frac{\partial X_{1}^{*}}{\partial \beta}-r \frac{\partial X_{2}^{*}}{\partial \theta}-\frac{\partial^{2}}{\partial \theta^{2}}\left(R_{2}^{*} X_{3}^{*}\right)=0
\end{aligned}
$$

To solve the dynamic problems of the pipeline section in question, it is necessary to obtain the equation of motion of the toroidal shell in displacements.

Therefore, we transform equation (5), expressing efforts $T_{1}$ and $M_{2}$ deformation $\varepsilon_{1}$ and $\theta$ in displacements, using the relationships between the forces, deformations and displacements of the semi muscular theory of shells, as well as expressions for the principal curvatures of the shell in the deformed state and for changing the curvature $\chi_{2}$ and torsion $\theta$ relations (3):

$$
\begin{aligned}
& T_{1}=\tilde{E} h \varepsilon_{1}=\tilde{E} h \frac{r}{R}\left(\frac{\partial u}{\partial \beta}+W_{y}\right), \\
& M_{2}=\tilde{D} \chi_{2}=\frac{\tilde{E} h^{3}}{12\left(1-v^{2}\right)}\left(-\frac{1}{r} \frac{\partial \vartheta}{\partial \theta}\right), \\
& W_{y}=w \cos \theta-v \sin \theta,
\end{aligned}
$$

where $u, v, w$ - referred to the radius $r$ dimensionless displacement components; $W_{y}$ - projection onto the axis $y$ Moving point A of the middle surface of the shell to the position $A^{*}$ as a result of deformation of its contour (see Figure 1); $\vartheta$-angle of rotation of the tangent to the midline of the section of the shell as a result of deformation of the cross section; $\tilde{E}$ - operator modulus of elasticity, which have the form $[17,18]$ :

$$
\tilde{E} \phi(t)=E_{01}\left[\phi(t)-\int_{0}^{t} R_{E}(t-\tau) \phi(t) d \tau\right]
$$

$\phi(t)$ - arbitrary time function; $R_{E}(t-\tau)$ - relaxation core; $E_{01}$ - instantaneous modulus of elasticity; We assume the 
integral terms in $(6$, a) to be small, then the functions $\phi(t)=\psi(t) e^{-i \omega_{R} t}$, where $\psi(t)$ - a slowly varying function of time, $\omega_{R}$ - real constant. Further, applying the freezing procedure [19], we note that the relations $(6$, a) are approximate of the form

$$
\bar{E} \phi=E\left[1-\Gamma^{C}\left(\omega_{R}\right)-i \Gamma^{S}\left(\omega_{R}\right)\right] \phi
$$

where $\Gamma^{C}\left(\omega_{R}\right)=\int_{0}^{\infty} R(\tau) \cos \omega_{R} \tau d \tau, \quad \Gamma^{S}\left(\omega_{R}\right)=\int_{0}^{\infty} R(\tau) \sin \omega_{R} \tau d \tau$, respectively, the cosine and sine Fourier images of the relaxation core of the material. As an example of a viscoelastic material, we take three parametric relaxation nuclei $R(t)=A e^{-\beta t} / t^{1-\alpha}$. On the influence function $R(t-\tau)$ the usual requirements of inerrability, continuity (except for $t=\tau$ ), sign-definiteness and monotony:

$$
R>0, \frac{d R(t)}{d t} \leq 0,0<\int_{0}^{\infty} R(t) d t<1 .
$$

$\vec{u}$ - vector of displacements of the environment of the $\mathrm{j}$-th layer.

Substituting relations (2), (3) and (6) into equation (5), neglecting here small nonlinear terms, we obtain the resolving equation of motion of the toroidal shell, expressed in displacements

$$
\begin{aligned}
& \frac{r^{2}}{R^{2}} \frac{\partial^{3} u}{\partial \beta \partial \theta^{2}} \cos \theta+\frac{r^{3}}{R^{3}} \frac{\partial^{3} u}{\partial \beta^{3}}-\frac{r^{2}}{R^{2}} \frac{\partial^{3} u}{\partial \beta^{3}}-\frac{r^{2}}{R^{2}} \frac{\partial}{\partial \theta}\left(\frac{\partial u}{\partial \beta} \sin \theta\right)+\frac{r^{3}}{R^{3}} \frac{\partial^{2} W_{y}}{\partial \beta^{2}}+ \\
& +\frac{r^{2}}{R^{2}} \frac{\partial}{\partial \theta}\left[\frac{\partial}{\partial \theta}\left(W_{y} \cos \theta\right)-W_{y} \sin \theta\right]+\frac{h^{2}}{r^{2} 12\left(1-v^{2}\right)} \frac{\partial^{3}}{\partial \theta^{3}}\left(\frac{\partial^{2} \vartheta}{\partial \theta^{2}}+\vartheta\right)= \\
& =-\frac{r^{2}}{E h R} \frac{\partial X_{1}^{*}}{\partial \beta}+\frac{r}{E h} \frac{\partial X_{2}^{*}}{\partial \theta}+\frac{r}{E h} \frac{\partial X_{2}^{*}}{\partial \theta}+\frac{r}{E h} \frac{\partial X_{2}^{*}}{\partial \theta}+\frac{r}{E h} \frac{\partial}{\partial \theta}\left(\frac{\partial^{2} \vartheta}{\partial \theta^{2}} X_{3}^{*}+\frac{\partial X_{3}^{*}}{\partial \theta}\right)
\end{aligned}
$$

where $X_{i}^{*}$ - components of inertia forces:

- tangential components in coordinates $\beta$ and $\theta$

$$
X_{1}^{*}=-r h p \frac{\partial^{2} u}{\partial t^{2}}, X_{2}^{*}=-r h p \frac{\partial^{2} v}{\partial t^{2}}
$$

- normal component along the normal to the middle surface of the shell

$$
X_{3}^{*}=-r h p \frac{\partial^{2} w}{\partial t^{2}}+p
$$

$p$ - internal pressure, including hydrodynamic, which occurs when the fluid moves, $p$ - density of the shell material.

The equation of motion of the toroidal shell (7) is a differential inhomogeneous partial differential equation with four unknown quantities $u, v, w, \vartheta$. Adding to it the three relations of the semi-membrane theory of shells:

$$
\frac{\partial v}{\partial \theta}+w=0, \frac{r}{R} \frac{\partial v}{\partial \beta}+\frac{\partial u}{\partial \theta}=0, \vartheta=\frac{\partial w}{\partial \theta}-v
$$

we obtain a complete system of equations with four unknowns. For a stationary fluid flow, the solution of equation (7), (8) allows us to determine the frequencies and shapes of the Eigen modes of the toroidal shell.

\section{Determination of Hydrodynamic Pressure Caused by a Fluid Flow}

One of the main factors that determine the solution of dynamic problems for pipelines with a flowing liquid is the hydrodynamic pressure of the liquid on the pipe wall. The curved section of the pipeline is considered as a toroidal shell with a radius of the cross-sectional line within which flows $U=$ const ideal incompressible fluid with density $p_{0}=$ const . The region bounded by a toroidal completely filled with a liquid is considered in toroidal coordinates $\alpha, \beta, \theta$, where $0 \leq \alpha \leq r$ - radial coordinate in the plane of the cross section of the torus (see Figure 1), $0 \leq \beta \leq \beta_{0}$ and $-\pi \leq \theta \leq \pi$. Lamé coefficients of coordinate surface $\alpha=$ const have the form [18]:

$$
H_{\alpha}=H_{\beta}=\frac{c}{\operatorname{ch} \alpha-\cos \beta}, H_{\theta}=\frac{c s h \alpha}{\operatorname{ch} \alpha-\cos \beta},
$$

where $c$ - scale factor.

The velocity field of an ideal incompressible fluid in the process of shell oscillation is an irrational potential field with a potential $\phi=\phi(\alpha, \beta, \theta, t)$. The system of basic equations of the potential flow of an ideal incompressible fluid includes [19]:

a. -equality of continuity (Laplace)

$$
\nabla^{2} \phi=0
$$

b. the equation of motion (Euler)

$$
\frac{\partial \phi}{\partial t}+Q(p)=0
$$

c. equation of state

$$
p_{0}=\text { const }
$$


where $Q(p)$ - the pressure function defined in $p_{0}=$ const equality

$$
Q(p)=\frac{1}{p_{0}}\left(p-p_{0}\right)
$$

where $p$ and $p_{0}$ - hydrodynamic and hydrostatic pressure, respectively.

From (10) - (13), a relationship is established between the hydrodynamic pressure $p$ and the potential of the disturbed velocities $\varphi$ :

$$
p=p_{0}-p_{0}\left(\frac{\partial \phi}{\partial t}+\frac{U}{R} \frac{\partial \phi}{\partial \beta}\right)
$$
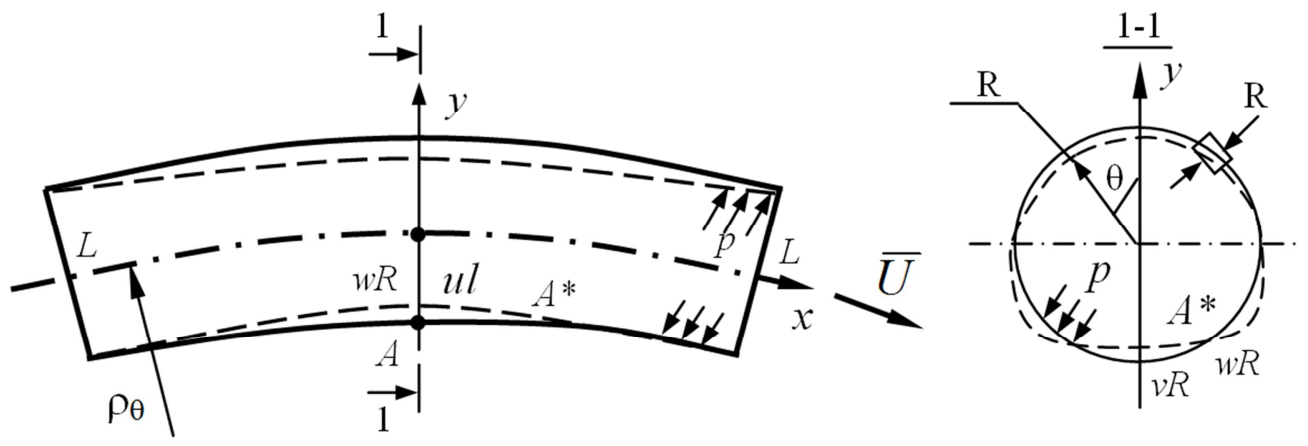

Figure 1. Pipeline section with flowing liquid.

Considering the velocity vector of the fluid flow $\bar{U}$ in toroidal coordinates, we write the expressions for its components by $\alpha, \beta, \theta$ :

$$
U_{\alpha}=\frac{1}{H_{\alpha}} \frac{\partial \phi}{\partial \alpha}, U_{\beta}=\frac{1}{H_{\beta}} \frac{\partial \phi}{\partial \beta}, U_{\theta}=\frac{1}{H_{\theta}} \frac{\partial \phi}{\partial \theta}
$$

For the component of the velocity vector $U_{\alpha}$, directed to the normals to the deformed shell surface, the smooth flow around this surface by the liquid flow must be satisfied [20]:

$$
\begin{aligned}
\left.U_{\alpha}\right|_{\alpha=r}=\left.\frac{1}{H_{a}} \frac{\partial \phi}{\partial \alpha}\right|_{\alpha=r} & =-\left.r\left(\frac{\partial w}{\partial t}+\frac{U}{H_{\beta}} \frac{\partial w}{\partial \beta}\right)\right|_{a=r}, \quad(16) \\
& \frac{\partial}{\partial \alpha}\left(\frac{\sin \alpha}{\operatorname{ch} \alpha-\cos \beta \partial \alpha}\right)+\frac{\partial}{\partial \beta}\left(\frac{\sin \alpha}{\operatorname{ch} \alpha-\cos \beta} \frac{\partial \phi}{\partial \beta}\right)+\frac{1}{(\operatorname{ch} \alpha-\cos \beta) \operatorname{sh} \alpha} \frac{\partial^{2} \phi}{\partial \theta^{2}}=0
\end{aligned}
$$

where $w$ - categorized as radius $r$ the dimensionless component of displacement of the points of the middle surface of the shell (Figure 1).

Thus, the problem of determining the hydrodynamic pressure of a liquid on the pipe wall reduces to finding the potential $\phi$, satisfying the Laplace equation (10) and conditions (14), (16) for $\alpha=r$.

The Laplace equation (10) in a toroidal coordinate system $\alpha, \beta$ and $\theta$ has the form:
As a result of the separation of variables after substitution

$$
\phi=(2 \operatorname{ch} \alpha-2 \cos \beta)^{1 / 2} \psi
$$

and representations of an unknown function $\psi(\alpha, \beta, \theta, t)$ in the form of:

$$
\psi=A(\alpha) B(\beta) C(\theta) \Phi(t)
$$

we obtain from (17) the known equation of the torus:

$$
A_{\alpha}^{\prime \prime}+\frac{\operatorname{ch} \alpha}{\operatorname{sh} \alpha} A_{\alpha}^{\prime}-\left[\left(n-\frac{1}{2}\right)^{2}+\frac{\mu^{2}}{\operatorname{sh}^{2} \alpha}\right] A=0
$$

where $\mu=$ const, $n=$ const .

The general solution of the torus equation (20) is determined by a linearly independent combination of torus functions $P_{n-\frac{1}{2}}(\operatorname{ch} \alpha)$ and $Q_{n-\frac{1}{2}}(\operatorname{ch} \alpha)$, representing one of the kinds of Legendre functions of the 1st and 2nd kind:

$$
A(\alpha)=A_{1} P_{n-\frac{1}{2}}(\operatorname{ch} \alpha)+A_{2} Q_{n-\frac{1}{2}}(\operatorname{ch} \alpha)
$$

Taking into account that in the problem posed, we consider the domain bounded by the surface of the torus by the coordinate $\alpha$, varying within $0 \leq \alpha \leq r$, and that when $\alpha \rightarrow 0$ Legendre function of the second kind $Q_{n-\frac{1}{2}}(c h r) \rightarrow \infty$, in the solution of (21) it is necessary to put $A_{2}=0$. Therefore, the solution of the equation of the torus (20) will be expressed only through the Legendre function of the first kind: 


$$
A(\alpha)=A_{1} P_{n-\frac{1}{2}}(\operatorname{ch} \alpha)
$$

and the solution of the Laplace equation (17) taking into account (18), (19), and (22) will have the form:

$$
\phi(\alpha, \beta, \theta, t)=(2 \operatorname{ch} \alpha-2 \cos \beta)^{1 / 2} P_{n-\frac{1}{2}}(\operatorname{ch} \alpha) A_{1} \zeta(\beta, \theta, t)
$$

Composition $A_{1} \zeta(\beta, \theta, t)$ we find from (16), taking the partial derivative $\left(\frac{\partial \phi}{\partial \alpha}\right)$. After substituting the value of this product in (23), we obtain an expression for the velocity potential:

$$
\phi=-\frac{r H_{\alpha}\left(\frac{\partial w}{\partial t}+\frac{U}{H_{\beta}} \frac{\partial w}{\partial \beta}\right) B^{1 / 2} P_{n-\frac{1}{2}}(c h r)}{B^{-1 / 2} \operatorname{shr} P_{n-\frac{1}{2}}(c h r)-B^{1 / 2} P_{n-\frac{1}{2}}^{\prime}(c h r)}
$$

where

$$
B=2(\operatorname{ch} r-\cos \beta)
$$

We find the hydrodynamic pressure of the flowing liquid on the wall of the shell from (14), neglecting small second- order ones arising in the calculation of the partial function $\phi$ by $\beta$ :

$$
p=p_{0}-p_{0} r H_{\alpha} \Phi_{n}\left[\frac{\partial^{2} w}{\partial t^{2}}+\left(\frac{1}{R}+\frac{1}{H_{\beta}}\right) \frac{\partial^{2} w}{\partial \beta \partial t} U+\frac{U^{2}}{R H_{\beta}} \frac{\partial^{2} w}{\partial \beta^{2}}\right],
$$

where is denoted

$$
\Phi_{n}=-\left(\frac{s h r}{B}+\frac{P_{n-\frac{1}{2}}^{\prime}(c h r)}{P_{n-\frac{1}{2}}(c h r)}\right)^{-1}
$$

In formula (26) for hydrodynamic pressure, the expression in parentheses, by analogy with the cylindrical [21], should be regarded as reduced acceleration (with allowance for the velocity $U$ ) element of the shell, and the value $p_{0} \Phi_{n}$, density-dependent $p_{0}$, be regarded as an adjoined mass of liquid.

\section{The Equation of Motion of Toroidal Shells with a Stationary Fluid Flow}

In toroidal coordinates $\beta, \theta$ the equation of motion of shells takes the form:

$$
\begin{aligned}
& \frac{r^{3}}{R^{3}} \frac{\partial^{2} W_{\alpha}}{\partial \beta^{2}} \cos \theta+\frac{r^{2}}{R^{2}} \frac{\partial^{3} u}{\partial \beta \partial \theta^{2}} \cos \theta+\frac{r^{3}}{R^{3}} \frac{\partial^{3} u}{\partial \beta^{3}}-\frac{r^{2}}{R^{2}} \cos \theta \frac{\partial u}{\partial \beta}-\frac{r^{2}}{R^{2}} \sin \theta \frac{\partial^{3} u}{\partial \theta \partial \beta}+ \\
& +\frac{r^{2}}{R^{2}} \frac{\partial}{\partial \theta^{2}}\left(W_{\alpha} \cos \theta\right)-\frac{\partial}{\partial \theta}\left(W_{\alpha} \sin \theta\right)+h_{\rho}^{e} \frac{\partial^{3}}{\partial \theta^{2}}\left(\frac{\partial^{2} \vartheta}{\partial \theta^{2}}+\delta\right)=\frac{r^{3}}{E R} \rho \frac{\partial^{3} u}{\partial t^{2} \partial \beta}- \\
& -r_{p} \frac{\partial^{3} V}{\partial \theta \partial t^{2}}+r_{p} \frac{\partial^{3} \vartheta}{\partial \theta^{3}}-r_{\rho} \frac{\partial^{u} w}{\partial \theta \partial t^{2}}+r_{E} \frac{\partial^{2}}{\partial \theta^{2}}\left(\rho_{\text {ж }}\right)
\end{aligned}
$$

where $u, V, w, W_{\alpha}, \vartheta$ - moving the shell in a toroidal coordinate;

$$
\begin{gathered}
h_{p}^{2}=\frac{h^{2}}{12 r^{2}\left(1-v^{2}\right)} ; \quad r_{p}=\frac{r^{2}}{E} \rho ; \quad r_{h}=\frac{r}{E h} p_{0} ; \quad r_{\rho}=\frac{r^{2}}{E} \rho ; \\
r_{E}=\frac{r^{2}}{E} ; \quad r_{E}^{*}=r^{3} \rho /(E R)
\end{gathered}
$$

Here, in the expression for the normal component of inertia forces $F_{3}{ }^{*}$ The internal pressure on the pipe wall in accordance with clause 2 is presented as a sum:

$$
p=p_{0}+p_{\varkappa}
$$

where $p_{0}=$ const - constant hydrostatic pressure; $p_{\varkappa}$ - the hydrodynamic pressure of the fluid flow in the curved section of the pipeline, determined through Legendre functions. The last term on the right-hand side of equation (28) contains the derivative of $\theta$ from a work $\frac{\partial^{2} \vartheta}{\partial \theta^{2}} F_{3}^{*}$. After differentiation, taking into account (29) and discarding the small nonlinear terms in Eq. (28), the following terms remain (the last three terms on the right-hand side)

$$
\frac{r}{E h} p_{0} \frac{\partial^{3} \vartheta}{\partial \theta^{3}}-\frac{r^{2}}{E} p \frac{\partial^{4} w}{\partial \theta^{2} \partial t^{2}}+\frac{r}{E h} \frac{\partial^{2}}{\partial \theta^{2}}\left(p_{\varkappa}\right)
$$

Adding to the equation of motion (28) the relationship of the semi muscular theory of shells and using formula (30) for the hydrodynamic pressure $p_{\varkappa}$, we obtain a complete system of equations for the problem in the displacements

$$
\begin{aligned}
& \frac{r^{2}}{R^{2}} \frac{\partial^{3} u}{\partial \beta \partial \theta^{2}} \cos \theta+\frac{r^{3}}{R^{3}} \frac{\partial^{3} u}{\partial \beta^{3}}-\frac{r^{2}}{R^{2}} \frac{\partial}{\partial \theta}\left(\frac{\partial u}{\partial \beta} \sin \theta\right)+\frac{r^{3}}{R^{3}} \frac{\partial^{2} W_{\alpha}}{\partial \beta^{2}}+ \\
& +\frac{r^{2}}{R^{2}} \frac{\partial}{\partial \theta}\left[\frac{\partial}{\partial \theta}\left(W_{\alpha} \cos \theta\right)-W_{\alpha} \sin \theta\right]+\frac{h_{p}^{2}}{1} \frac{\partial^{3}}{\partial \theta^{3}}\left(\frac{\partial^{2} \vartheta}{\partial \theta^{2}}+\vartheta\right)= \\
& =r_{E} \frac{\partial}{\partial \beta}\left(\frac{\partial^{2} u}{\partial t^{2}}\right)-\frac{r^{2}}{E} p \frac{\partial}{\partial \theta}\left(\frac{\partial^{2} V}{\partial t^{2}}\right)+r_{n} \frac{\partial^{3} \vartheta}{\partial \theta^{3}}-r_{p} \frac{\partial^{4} w}{\partial \theta^{2} \partial t^{2}}- \\
& -r_{\varphi}\left(\frac{\partial^{4} w}{\partial \theta^{2} \partial t^{2}}+\frac{u^{2}}{R r} \frac{\partial^{4} w}{\partial \theta^{2} \partial \beta^{2}}\right) ;
\end{aligned}
$$


where $r_{\varphi}=\frac{r^{2} p_{0} \phi_{n}}{E h}$.

It should be noted that the displacement components $u, v, w$ (30), (31) are also dimensionless. The boundary conditions can be as follows.

1. Both ends are hinged. The boundary conditions corresponding to this fixation can be formulated as follows:

at $\beta=0 u \quad \beta=\alpha \quad w=0 ; v=0 ; T_{1}=0 ; M_{1}=0$.

These conditions, expressed in functions $f_{n}(\beta)$, have the form:

at

$\beta=0 \quad$ и $\beta=\alpha \quad f_{n}(0)=f_{n}(\alpha)=0 ; f_{n}^{\prime \prime}(0)=f_{n}^{\prime \prime}(\alpha)=0$.

This fastening corresponds to a fundamental beam function:

$$
f_{n}(\beta)=\sin \frac{\lambda_{n} \beta}{\alpha}, \quad \lambda_{n}=n \pi
$$

2. Hard pinch on the ends. With such a fixation of both ends, the boundary conditions have the form:

at $\beta=0 u \quad \beta=\alpha \quad u=0 ; v=0 ; w=0 ; \vartheta=0$.

From this follows:

at

$\beta=0 \quad$ и $\beta=\alpha \quad f_{n}(0)=f_{n}(\alpha)=0 ; \quad f_{n}^{\prime}(0)=f_{n}^{\prime}(\alpha)=0$.

The fundamental functions corresponding to a given fixation have the form:

$$
\begin{gathered}
f_{n}(\beta)=\sin \frac{\lambda_{n} \beta}{\alpha}-\operatorname{sh} \frac{\lambda_{n} \beta}{\alpha}-g_{n}\left(\cos \frac{\lambda_{n} \beta}{\alpha}-\operatorname{ch} \frac{\lambda_{n} \beta}{\alpha}\right), \\
g_{n}=\frac{\operatorname{sh} \lambda_{n}-\sin \lambda_{n}}{\operatorname{ch} \lambda_{n}-\cos \lambda_{n}} \\
\lambda_{1}=4,730, \quad \lambda_{2}=7,8532, \quad \lambda_{n}=\frac{2 n+1}{4} \pi(n>2) .
\end{gathered}
$$

To solve the system of equations (30), (31), we represent the normal component of the displacement arising when the toroidal shell flexures $w(\beta, \theta, t)$ in a form that satisfies the boundary conditions at the edges of the shell:

$$
\left.w\right|_{\substack{\beta=0 \\ \beta=\pi}}=0,\left.\quad \frac{\partial^{2} w}{\partial \beta^{2}}\right|_{\substack{\beta=0 \\ \beta=\pi}}=0
$$

And also satisfying the cyclicity conditions along the circumferential coordinate $\theta$ :

$$
w(\beta, \theta, t)=f(t) a_{m} \cos m \theta \sin n \beta
$$

where $f(t)$ - time function $t, a_{m}=$ const, $m, n$ - wave numbers that determine the shape of the shell oscillations in the circumferential and longitudinal directions, respectively.

From the relations (33) between the components of displacement at a value $w$ by (31) we obtain expressions for the remaining components of the displacement and the angle of rotation:

$$
\begin{aligned}
& u=-\frac{r}{R} \frac{n}{m^{2}} f(t) a_{m} \cos m \theta \cos n \beta, v=-\frac{1}{m} f(t) a_{m} \sin m \theta \sin n \beta, \\
& \vartheta=-\frac{m^{2}-1}{m} f(t) a_{m} \sin m \theta \sin n \beta, W_{\alpha}=\frac{1}{2}\left(a_{m+1} \frac{m+2}{m+1}+a_{m-1} \frac{m-2}{m-1}\right) \cos m \theta \sin n \beta .
\end{aligned}
$$

Substituting expressions (33), (34) for the displacement components and the rotation angle into the equation of motion of the shell (34) and calculating the partial derivatives with respect to $\beta$ and $\theta$, we obtain a resolving equation with respect to unknown amplitude values $a_{m}$ and containing the function of time $f(t)$ and its second time derivative $f^{\prime \prime}(t)$ :

$$
\begin{aligned}
& f^{\prime \prime}(t)\left(\frac{r^{4}}{E R^{2}} p b_{m} \frac{n^{2}}{m^{3}} \sin m \theta+\frac{r^{2}}{E} p b_{m} \frac{1}{m} \sin m \theta+\frac{r^{2}}{E h} p_{0} \Phi_{n}^{*} b_{m} \sin m \theta\right)= \\
& f(t)\left\{-\frac{r^{4}}{R^{4}} \frac{n^{4}}{m^{3}} a_{m} \sin m \theta+\frac{r^{3}}{2 R^{3}} \frac{n^{2}}{m} a_{m}(\sin (m-1) \theta+\sin (m+1) \theta)+\right. \\
& +\frac{r^{3}}{2 R^{3}} \frac{n^{2}}{m}\left(a_{m+1} \frac{m+2}{m+1}+b_{m-1} \frac{m-2}{m-1}\right) \sin m \theta-\frac{r^{2}}{R^{2}}\left(a_{m+1} \frac{m+2}{m+1}+b_{m-1} \frac{m-2}{m-1}\right) \times \\
& \times\left(\frac{m-2}{4} \sin (m-1) \theta+\frac{m+2}{4} \sin (m+1)\right) \theta- \\
& \left.-\left(h_{p}{ }^{2}\left[m\left(m^{2}-1\right) \times\left(m^{2}-1\right)+\frac{r^{3} 12\left(1-v^{2}\right)}{E h^{3}} p_{0}\right] \sin m \theta+\frac{r^{3}}{E h} p_{0} b_{m} \Phi_{n}^{*} \frac{U^{2}}{R r} n^{2} m\right) \sin m \theta\right\}
\end{aligned}
$$

To simplify the form of equation (35), we introduce the we get: dimensionless shell thickness parameter $h_{v}$ : $h_{v}=\frac{h}{r c_{v}}, c_{v}=\sqrt{12\left(1-v^{2}\right)}, v$ - Poisson's ratio.

$$
f(t) \Omega_{m 1}-f^{\prime}(t) \Omega_{m 2}=0
$$

We divide each term of equation (35) by $h_{v}^{2}$. As a result,

where 


$$
\begin{aligned}
& \Omega_{m 1}=\left\{-\frac{r^{4}}{R^{4} h_{v}^{2}} \cdot \frac{n^{4}}{m^{3}} a_{m} \sin m \theta+\frac{r^{3}}{2 R^{3} h_{v}^{2}} \frac{n^{2}}{m} .\right. \\
& \cdot\left[a_{m}(\sin (m-1) \theta+\sin (m+1) \theta)+\left(a_{m+1} \frac{m+2}{m+1}+a_{m-1} \frac{m-2}{m-1}\right) \sin m \theta\right]- \\
& -\frac{r^{2}}{2 R^{2} h_{v}^{2}}\left(a_{m+1} \frac{m+2}{m+1}+a_{m-1} \frac{m-2}{m-1}\right) \times((m-2) \sin (m-1) \theta+(m+2) \sin (m+1) \theta)-m\left(m^{2}-1\right) . \\
& \cdot\left(m^{2}-1+\frac{r}{E h h_{v}^{2}} p_{0}\right) \times b_{m} \sin m \theta+\frac{r}{R} \frac{r}{E h h_{v}^{2}} p_{0} \Phi_{n}^{*} U^{2} m n^{2} b_{m} \sin m \theta ; \\
& \Omega_{2 m}=\left[\frac{r}{E h h_{v}^{2}} p\left(\frac{r^{2}}{R^{2}} \frac{n^{2}}{m^{3}}+m+\frac{1}{m}\right)+r^{2} \frac{r}{E h h_{v}^{2}} p_{0} \Phi_{n}^{*} m\right] a_{m} \sin m \theta
\end{aligned}
$$

To simplify (36), we introduce the following notation:

$$
\rho^{*}=\frac{r}{E h h_{v}^{2}} p, \quad \rho_{0}^{*}=\frac{r}{E h h_{v}^{2}} p_{0}, \quad p_{0}^{*}=\frac{r}{E h h_{v}^{2}} p_{0}
$$

In addition, we transform equation (36) using the curvature parameter of the toroidal shell $\mu$, which characterizes not only the geometry of the shell, but also its material, since it includes the Poisson's ratio:

$$
\begin{aligned}
& \mu=\frac{r^{2}}{R h} c_{v}, \quad c_{v}^{2}=12\left(1-v^{2}\right) \\
&-\frac{1}{4} \mu^{2}\left(a_{m+1} \frac{m+2}{m+1}+b_{m-1} \frac{m-2}{m-1}\right)((m+2) \sin (m+1) \theta)-m\left(m^{2}-1\right)\left(m^{2}-1+p_{0}^{*}\right) a_{m} \sin m \theta+ \\
&\left.+\mu h_{v} p_{0}^{*} \Phi_{n}^{*} u^{2} m n^{2} a_{m} \sin m \theta\right\}+\sin \omega t\left[\frac{r h p^{*}}{m}\left(m^{2}-1\right)+r^{2} p_{0}^{*} \Phi_{n}^{*} m\right] \omega^{2} d_{m} \sin m \theta--d_{m} \sin \omega t \times \\
& \times\left\{-\mu^{4} h_{v}^{2} \frac{n^{4}}{m^{3}} a_{m} \sin m \theta+\frac{1}{2} \mu^{3} h_{v} \frac{n^{2}}{m} \cdot\left[a_{m}(\sin (m-1) \theta+\sin (m+1) \theta)+\left(a_{m+1} \frac{m+2}{m+1}+b_{m-1} \frac{m-2}{m-1}\right) \sin m \theta\right]=0\right.
\end{aligned}
$$

Here it is taken into account that in the penultimate term of equation (31) the quantity $\frac{r^{2}}{R^{2}}$, small in comparison with

Equating in (39) the factors for the same trigonometric functions $\sin \omega t$, we finally get wave number $m=1,2,3 \ldots$.

$$
\begin{aligned}
& \mu^{4} h_{v}^{2} \frac{n^{4}}{m^{3}} a_{m} \sin m \theta-\frac{\mu^{3} h_{v}}{2} \frac{n^{2}}{m} \times \\
& \times\left[a_{m}(\sin (m-1) \theta+\sin (m+1) \theta)+\left(a_{m+1} \frac{m+2}{m+1}+a_{m-1} \frac{m-2}{m-1}\right) \sin m \theta\right]+ \\
& \frac{\mu^{2}}{4} \times\left(a_{m+1} \frac{m+2}{m+1}+a_{m-1} \frac{m-2}{m-1}\right)((m-2) \sin (-1) \theta+(m+2) \sin (m+1) \theta)+ \\
& +m\left(m^{2}-1\right)\left(m^{2}-1+p_{0}^{*}\right) a_{m} \sin m \theta- \\
& -\mu h_{v} p_{0}^{*} \Phi_{n}^{*} u^{2} m n^{2} a_{m} \times \sin m \theta-\left(\frac{r h p^{*}}{m}\left(m^{2}+1\right)+r^{2} p_{0}^{*} \Phi_{n}^{*} m\right) a_{m} \omega^{2} \sin m \theta=0
\end{aligned}
$$

The dynamic equation of the motion of a toroidal shell with a stationary fluid flow (40), obtained on the basis of a geometrically nonlinear version of the semi-shell theory of shells and the theory of the potential flow of an ideal incompressible fluid, is a homogeneous equation. All terms of which are multipliers with trigonometric functions $\sin m \theta, \quad m=1,2,3 \ldots$, describing the deformation of the cross sections of the shell under bending vibrations. 


\section{Numerical Natural Oscillations of a Toroidal Shell with a Fluid Flow}

The study of the frequencies of the proper bending oscillations of the curved sections of pipelines (steel) with a steady flow of liquid is carried out numerically [22]. As the relaxation nucleus of a viscoelastic material, we take a threeparameter core $R(t)=\frac{A e^{-\beta t}}{t^{1-\alpha}}$ Rizhaniten-Koltunov [17], which has a weak singularity, where $A, \alpha, \beta$ - parameters materials [17]. We take the following parameters: $A=0,048 ; \beta=0,05 ; \quad \alpha=0,1$. Using the complex representation for the elastic modulus, described earlier. The roots of the frequency equation are solved by the Mueller method, at each iteration of the Muller method is applied by the Gauss method with the separation of the main element. Thus, the solution of equation (31) does not require the disclosure of the determinant. As the initial approximation, we choose the phase velocities of the waves of the elastic system. In the pipeline, water flows at a velocity u from 0 to $50 \frac{\mathcal{M}}{\mathrm{c}}$. The acquisition of the results allowed us to estimate the influence of the flow velocity on the frequencies of the first four waveforms ( $m=1,2,3,4$ at $n=1,2,3)$. Calculations were carried out for curvilinear pipes with relative thicknesses $\frac{h}{r}=\frac{1}{30}, \frac{1}{40}, \frac{1}{60}$ and different curvatures $\frac{r}{R}=\frac{1}{10}, \frac{1}{20}$, which corresponded to the parameters of curvature $\mu=5.8 ; 11.6$ and 23.1. These parameters, in turn, corresponded to the following values of the curvature of the bends and bends of the pipelines: $\lambda=0.57 ; 0.28$ and 0.14 the modulus of elasticity of the steel from which the pipes are made is assumed to be equal $E=2 \cdot 10^{5} \mathrm{MPa}$, Poisson's ratio $v=0.3$. The results of the calculations are presented in Tables 1-4 and in the graphs of Figs 2-4, which shows the change in the frequencies of the intrinsic bending vibrations $\omega_{m n}$ curvilinear sections of the steel pipeline, depending on the speed of the flowing liquid for different values of the shell thickness. The flow rate $u$, which varies in the range of actual velocities flowing in the fluid pipelines (up to $25 \frac{\mathrm{m}}{\mathrm{s}}$ ), little effect on the frequencies of natural oscillations of the curvilinear sections of the steel pipeline for all the investigated shell forms of oscillations ( $m=1,2,3,4$ при $n=1,2,3)$. Oscillation frequencies $\omega_{m n}$ decrease with an increase in the flow rate from 0 to $25 \frac{\mathrm{m}}{\mathrm{s}}$ not more than $10 \%$. For each of the sections of the pipeline considered, the largest frequencies of natural oscillations are in the first form $\omega_{1 n}$ at $m=1$.

Table 1. Natural frequencies as a function of the velocity of the flowing liquid.

\begin{tabular}{|c|c|c|c|c|}
\hline$\frac{r}{R}=\frac{1}{20}, \frac{h}{r}=\frac{1}{60}$ & & \multicolumn{2}{|c|}{$\omega_{m n}(\mathrm{~Hz})$ at the velocity of the flowing liquid in } & \multirow[b]{2}{*}{$u=40$} \\
\hline Form of oscillation & Frequencies & $u=0$ & $u=\mathbf{2 0}$ & \\
\hline \multirow{3}{*}{$m=1$} & $\omega_{11}$ & 26.46 & 21.01 & 17.25 \\
\hline & $\omega_{12}$ & 21.01 & 20.45 & 17.74 \\
\hline & $\omega_{13}$ & 22.92 & 22.72 & 20.55 \\
\hline \multirow{3}{*}{$m=2$} & $\omega_{21}$ & 13.39 & 12.83 & 10.42 \\
\hline & $\omega_{22}$ & 16.67 & 15.82 & 12.51 \\
\hline & $\omega_{23}$ & 18.68 & 18.44 & 16.20 \\
\hline \multirow{3}{*}{$m=3$} & $\omega_{31}$ & 13.02 & 12.29 & 9.28 \\
\hline & $\omega_{32}$ & 16.43 & 15.61 & 12.32 \\
\hline & $\omega_{33}$ & 18.34 & 18.17 & 15.83 \\
\hline \multirow{3}{*}{$m=4$} & $\omega_{41}$ & 19.47 & 19.33 & 14.21 \\
\hline & $\omega_{42}$ & 20.12 & 20.06 & 12.97 \\
\hline & $\omega_{43}$ & 21.36 & 21.22 & 11.38 \\
\hline
\end{tabular}

If there is no deformation of the contour of the cross sections of the pipe - that is, the pipe oscillates like a beam of tubular section. For the dynamic calculation of the pipeline, the most important is the shell mode (with $m=2$ and 3), corresponding to the deformed contour of the pipe crosssection. With the increase in the curvature of the pipeline section, that is, the ratio $\frac{r}{R}$, corresponding to the deformed contour of the pipe cross-section. With the increase in the curvature of the pipeline section, that is, the ratio
( $\frac{h}{r}=$ const $)$ frequencies $\omega_{m n}$ of their own flexural vibrations increase. Similarly, with an increase in the relative thickness $\left(\frac{h}{r}\right.$, at a constant curvature of the tube), the natural frequencies of bending vibrations increase. Thus, the greater the curvature of the tube, the more rigid it becomes, and the thicker the pipe wall, the more rigid it is. This is also seen from the graphs in Figure 6, which shows monotonically increasing frequency dependence $\omega_{21}$ in the form of oscillation. 
Table 2. Eigen frequencies depending on the velocity of the flowing liquid.

\begin{tabular}{|c|c|c|c|c|}
\hline \multirow{2}{*}{$\begin{array}{l}\frac{\boldsymbol{r}}{\boldsymbol{R}}=\frac{1}{20}, \frac{\boldsymbol{h}}{\boldsymbol{r}}=\frac{1}{60} \quad \boldsymbol{\mu}=11,5 \\
\text { Form of oscillation }\end{array}$} & & \multicolumn{2}{|c|}{$\omega_{m n}(\mathrm{~Hz})$ at the velocity of the flowing liquid in } & $\frac{m}{s}$ \\
\hline & Frequencies & $u=0$ & $u=20$ & $u=40$ \\
\hline \multirow{3}{*}{$m=1$} & $\omega_{11}$ & 55.34 & 53.47 & 51.13 \\
\hline & $\omega_{12}$ & 56.05 & 55.27 & 52.39 \\
\hline & $\omega_{13}$ & 60.56 & 59.99 & 57.18 \\
\hline \multirow{3}{*}{$m=2$} & $\omega_{21}$ & 36.26 & 34.09 & 28.57 \\
\hline & $\omega_{22}$ & 44.60 & 43.78 & 40.35 \\
\hline & $\omega_{23}$ & 51.67 & 50.52 & 47.06 \\
\hline \multirow{3}{*}{$m=3$} & $\omega_{31}$ & 35.02 & 33.01 & 26.51 \\
\hline & $\omega_{32}$ & 43.11 & 43.50 & 39.22 \\
\hline & $\omega_{33}$ & 50.03 & 49.63 & 46.58 \\
\hline \multirow{3}{*}{$m=4$} & $\omega_{41}$ & 53.01 & 50.31 & 47.44 \\
\hline & $\omega_{42}$ & 54.95 & 52.05 & 48.50 \\
\hline & $\omega_{43}$ & 55.82 & 53.92 & 49.99 \\
\hline
\end{tabular}

At $m=2$ the curvature parameter of the pipeline section $\mu$ and $\frac{h}{r}$ significantly affects the natural frequencies of oscillations.

The smaller the curvature of the tube and the thinner its walls, the lower its frequencies of natural oscillations $\omega_{m n}$ practically in all forms.

At the speed of the liquid $40 \leq u \leq 50$ the minimum value of the frequencies $\omega_{m n}$. At these values of the frequencies of the curvilinear shells, the amplitudes and deformations take on the maximum values

Study of frequencies $\omega_{m n}$ for the first three forms of natural oscillations $m=1,2,3$ curvilinear sections of polyethylene pipelines with a much smaller modulus of elasticity than in steel pipes made it possible to reveal a significant dependence of the values of the oscillation frequencies on the flow velocity of the liquid. Low natural frequencies of pipelines are dangerous due to the possibility of occurrence of a resonant situation.

Table 3. Eigen frequencies depending on the velocity of the flowing liquid

\begin{tabular}{|c|c|c|c|c|}
\hline$\frac{r}{R}=\frac{1}{20}, \frac{h}{r}=\frac{1}{60}$ & & \multicolumn{2}{|c|}{$\omega_{m n}(\mathrm{~Hz})$ at the velocity of the flowing liquid in $\frac{n}{\mathrm{~s}}$} & \\
\hline Form of oscillation & Frequencies & $u=0$ & $u=\mathbf{2 0}$ & $u=40$ \\
\hline \multirow{3}{*}{$m=1$} & $\omega_{11}$ & 8.61 & 8.01 & 7.42 \\
\hline & $\omega_{12}$ & 10.52 & 10.23 & 9.22 \\
\hline & $\omega_{13}$ & 12.25 & 12.03 & 11.93 \\
\hline \multirow{3}{*}{$m=2$} & $\omega_{21}$ & 8.30 & 7.83 & 7.12 \\
\hline & $\omega_{22}$ & 10.23 & 9.84 & 9.01 \\
\hline & $\omega_{23}$ & 11.84 & 11.75 & 11.52 \\
\hline \multirow{3}{*}{$m=3$} & $\omega_{31}$ & 7.13 & 6.70 & 6.32 \\
\hline & $\omega_{32}$ & 8.82 & 8.42 & 7.82 \\
\hline & $\omega_{33}$ & 10.12 & 9.91 & 9.72 \\
\hline \multirow{3}{*}{$m=4$} & $\omega_{41}$ & 11.13 & 11.05 & 10.11 \\
\hline & $\omega_{42}$ & 12.42 & 12.72 & 12.92 \\
\hline & $\omega_{43}$ & 14.03 & 13.79 & 13.25 \\
\hline
\end{tabular}

Table 4. Natural frequencies as a function of the velocity of the flowing liquid.

\begin{tabular}{lllll}
\hline$\frac{\boldsymbol{r}}{\boldsymbol{R}}=\frac{1}{20}, \frac{\boldsymbol{h}}{\boldsymbol{r}}=\frac{1}{60} \boldsymbol{\mu}=5,5$ & & $\boldsymbol{\omega}_{\boldsymbol{m} \boldsymbol{n}}(\mathrm{Hz})$ at the velocity of the flowing liquid in $\frac{\boldsymbol{m}}{\boldsymbol{s}}$ & \\
\hline Form of oscillation & Frequencies & $\boldsymbol{u}=\mathbf{0}$ & $\boldsymbol{u}=\mathbf{2 0}$ & $\boldsymbol{u}=\mathbf{5 0}$ \\
\hline \multirow{2}{*}{$m=1$} & $\omega_{11}$ & 31.21 & 30.52 & 29.09 \\
& $\omega_{12}$ & 40.02 & 39.31 & 38.05 \\
\hline
\end{tabular}




\begin{tabular}{|c|c|c|c|c|}
\hline \multirow{2}{*}{$\begin{array}{l}\frac{\boldsymbol{r}}{\boldsymbol{R}}=\frac{1}{20}, \frac{\boldsymbol{h}}{\boldsymbol{r}}=\frac{1}{60} \quad \boldsymbol{\mu}=5,5 \\
\text { Form of oscillation }\end{array}$} & \multicolumn{4}{|c|}{$\omega_{m n}(\mathrm{~Hz})$ at the velocity of the flowing liquid in $\frac{m}{s}$} \\
\hline & Frequencies & $u=0$ & $u=20$ & $u=50$ \\
\hline \multirow{4}{*}{$m=2$} & $\omega_{13}$ & 47.02 & 46.64 & 46.18 \\
\hline & $\omega_{21}$ & 24.24 & 53.02 & 20.39 \\
\hline & $\omega_{22}$ & 30.56 & 29.26 & 26.55 \\
\hline & $\omega_{23}$ & 36.44 & 35.91 & 35.27 \\
\hline \multirow{3}{*}{$m=3$} & $\omega_{31}$ & 20.83 & 19.51 & 16.17 \\
\hline & $\omega_{32}$ & 24.91 & 24.01 & 23.34 \\
\hline & $\omega_{33}$ & 28.61 & 28.39 & 28.35 \\
\hline \multirow{3}{*}{$m=4$} & $\omega_{41}$ & 29.02 & 28.92 & 28.98 \\
\hline & $\omega_{42}$ & 30.17 & 29.15 & 29.01 \\
\hline & $\omega_{43}$ & 31.36 & 30.97 & 30.05 \\
\hline
\end{tabular}

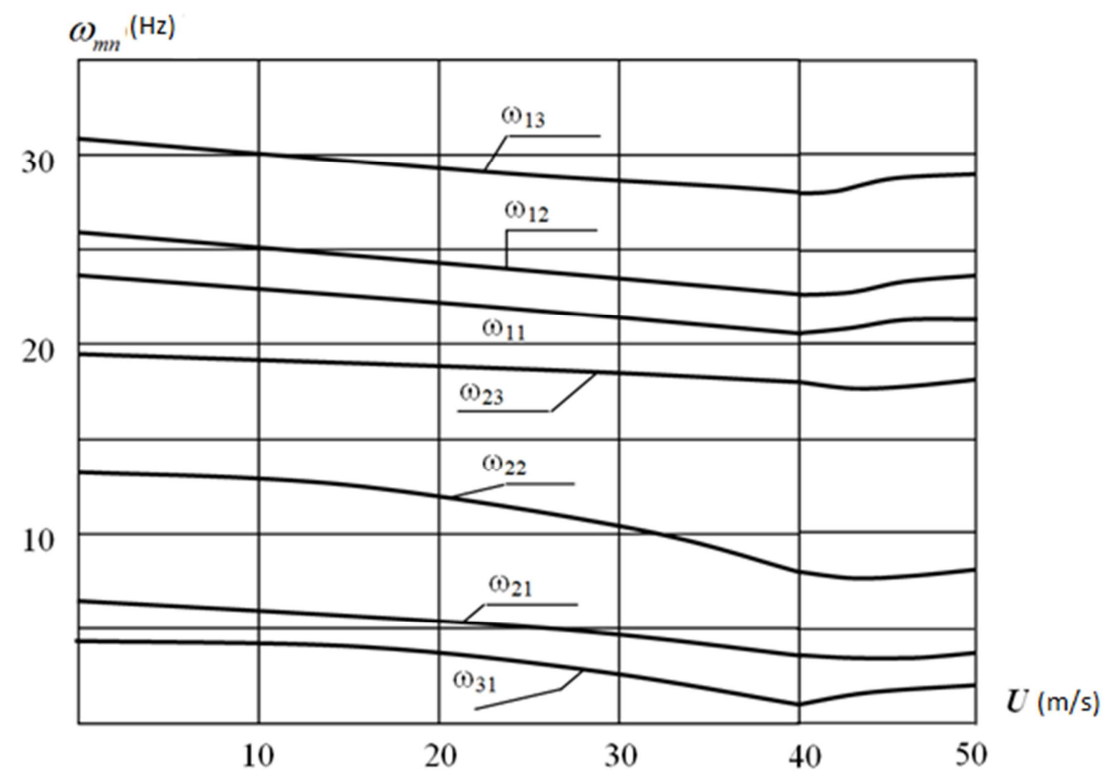

Figure 2. The change in the frequencies of the intrinsic bending vibrations from the velocity of the flowing liquid $(h=0.001)$.

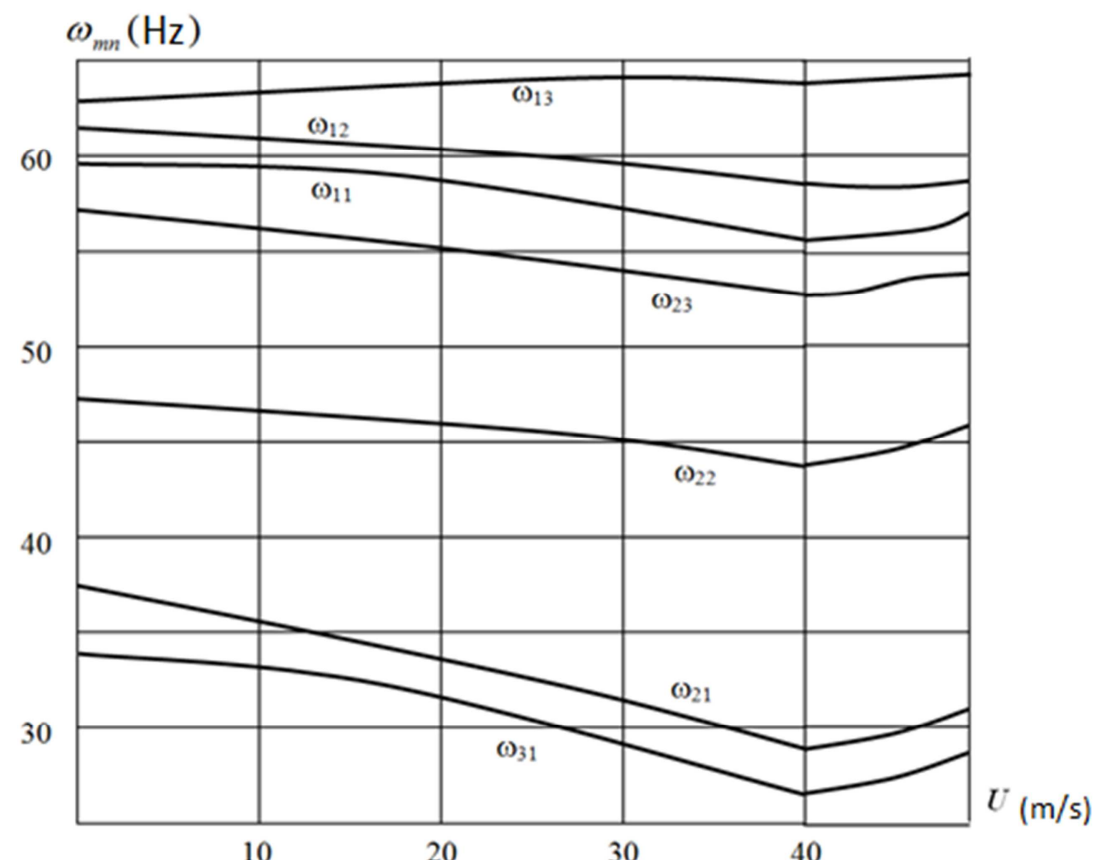


Figure 3. Change in the frequencies of natural flexural vibrations from the velocity of the flowing fluid $(h=0.005)$.

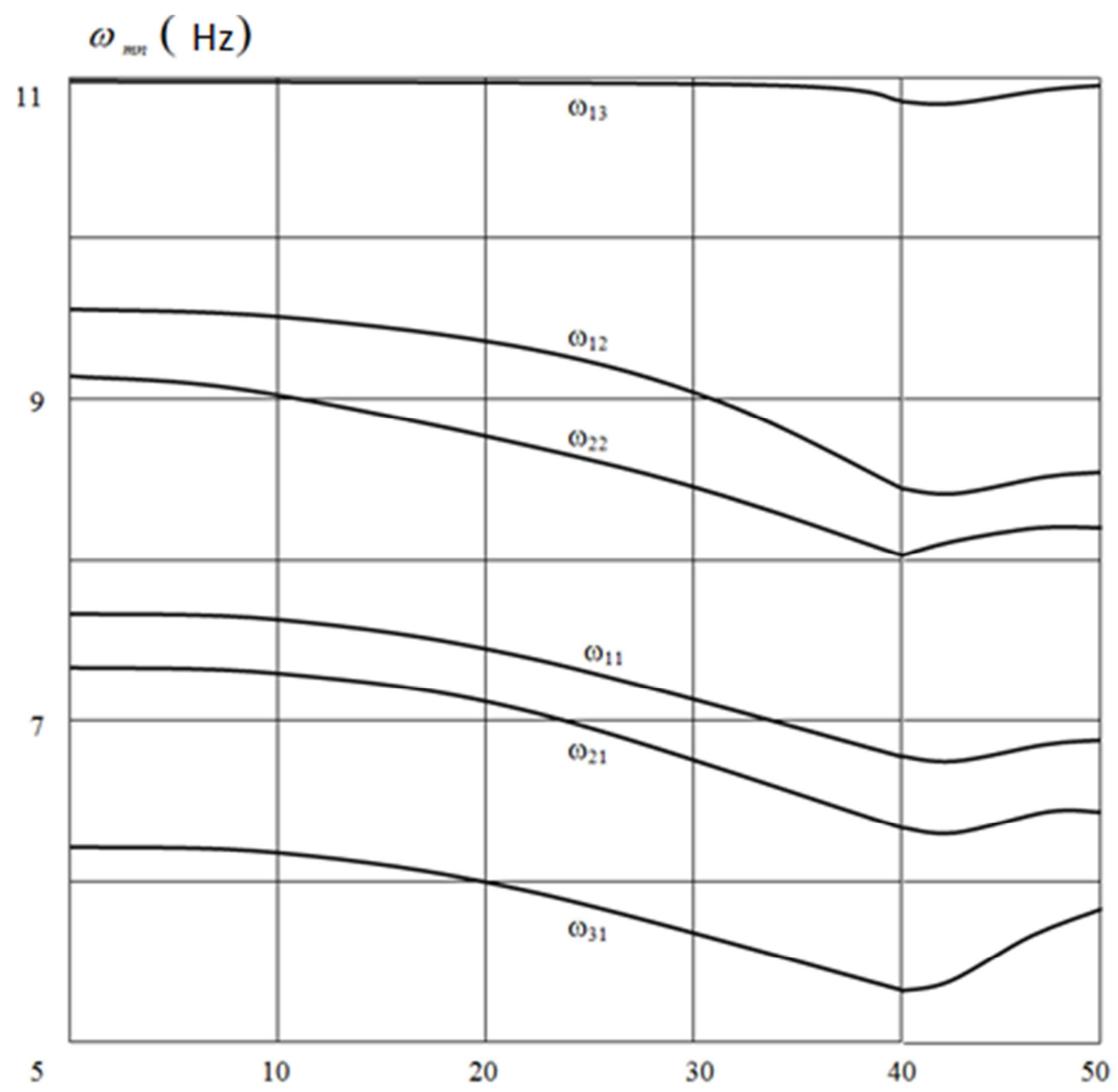

Figure 4. Changing the frequencies of own flexural vibrations from the velocity of the flowing fluid ( $h=0.01$ ).

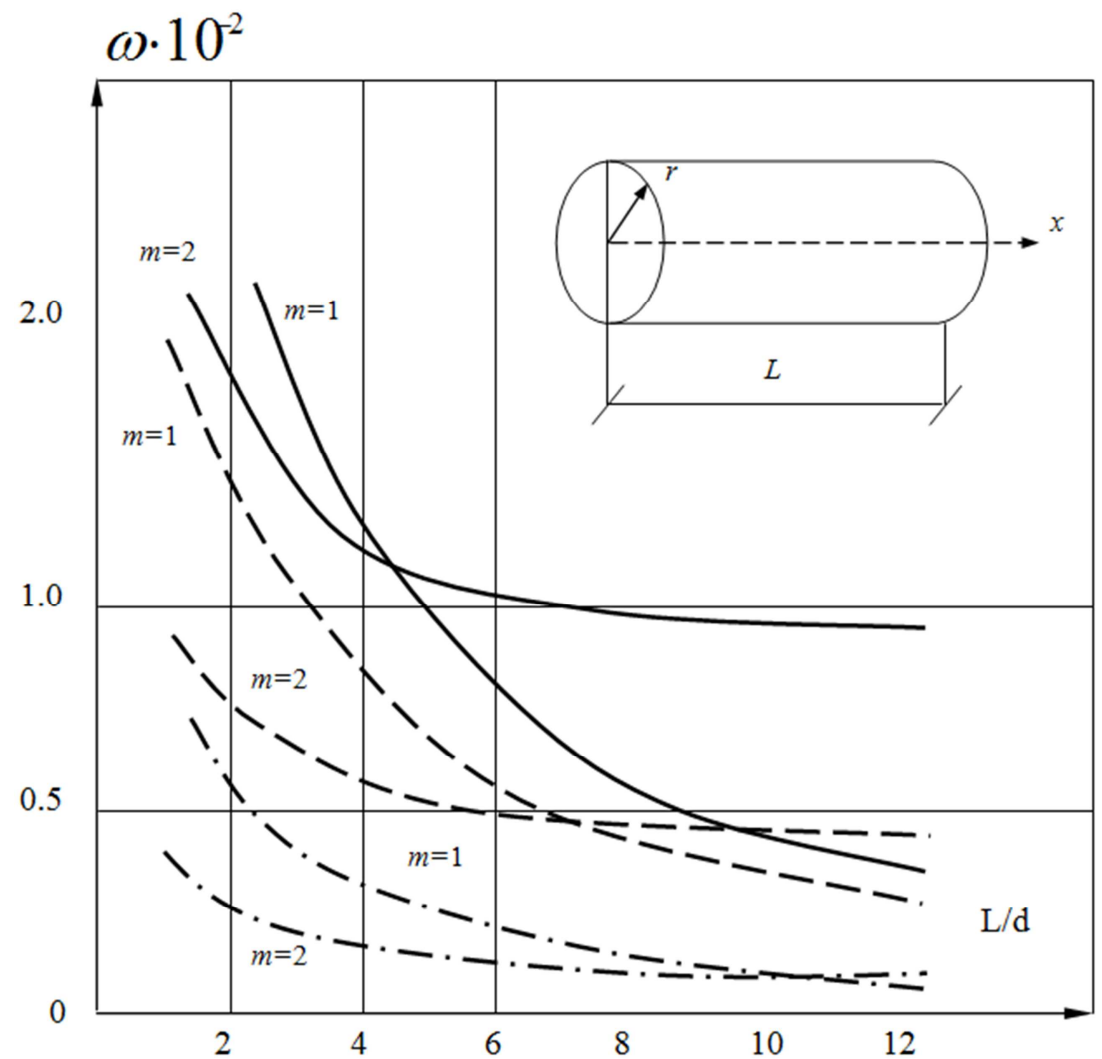


Figure 5. Dependence of the frequency of free oscillations on $L / d$.

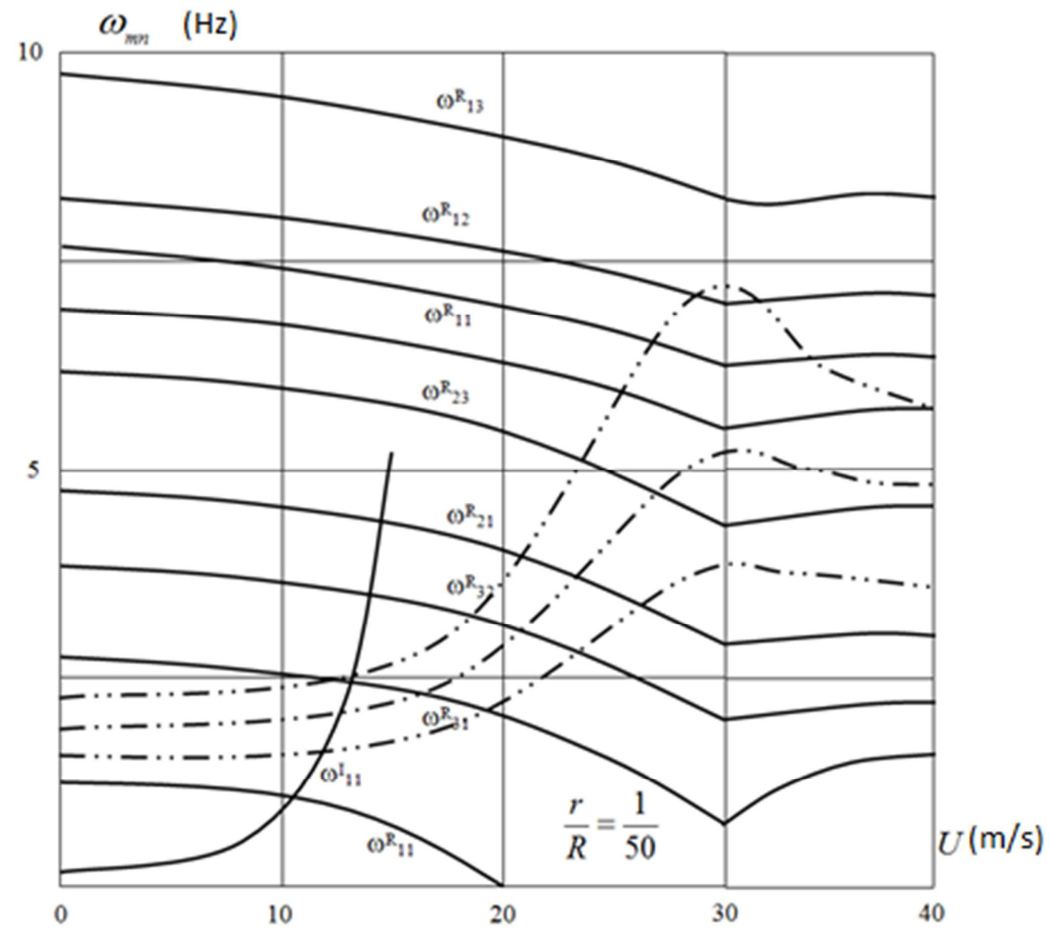

Figure 6. Changing the frequencies of own bending vibrations from the flowing fluid velocity.

Thus, when calculating the curvilinear section of a polyethylene pipeline with relative curvature $\frac{r}{R}=\frac{1}{50}$ already according to the first form of oscillation $(m, n=1)$ at a liquid flow rate $U=20 \frac{\mathrm{m}}{\mathrm{s}}$ frequency $\omega_{11}=0$ (see the diagram of the dashed line in Figure 6). It means that for such a pipeline speed $U=20 \frac{m}{s}$ is critical and it has lost stability.

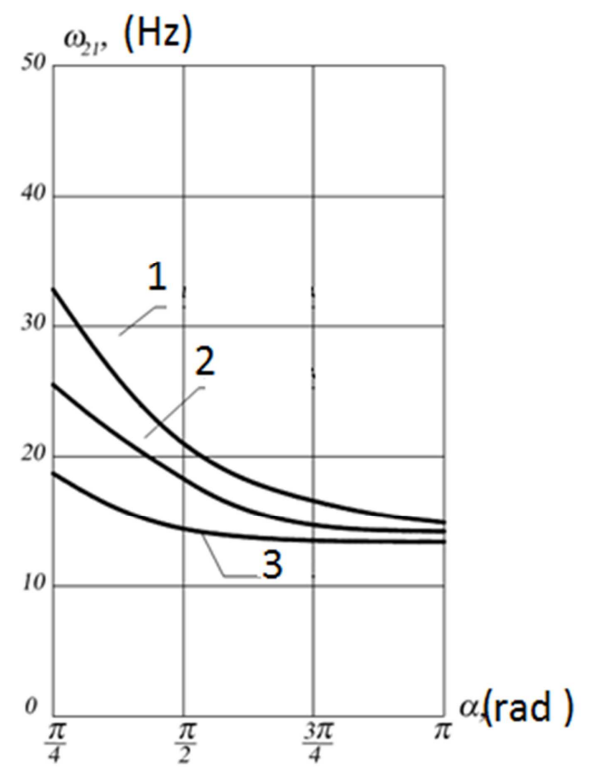

Figure 7. Dependence $\omega_{21}$ from the corner $\alpha$ at a relative thickness $h / r=1 / 72$ with different types of fixing the end cross sections for $\mu=18$.(1-jamming- jamming; 2-articulated- jamming;3-articulated-articulated)

Figure 7 shows the change in the real parts of the natural frequency from $\alpha$ various fastenings [23, 24].

\section{Conclusions}

1. The problem posed for the natural oscillations of thinwalled toroidal shells with a flowing liquid is solved on the basis of a geometrically nonlinear semimuscular theory of shells in toroidal coordinates. The hydrodynamic pressure of the liquid flow is obtained using the theory of the potential flow of a fluid. The obtained solution makes it possible to determine the values of the natural oscillation frequencies of the pipeline according to shell wave numbers $m, n=1,2,3, \ldots$.

2 . Based on the general solution of the problem, a method for determining the natural oscillation frequencies of toroidal shells of large diameter under the influence of internal hydrostatic pressure and hydrostatic pressure caused by fluid motion has been developed. The application of this technique in dynamic calculations of pipelines will avoid the occurrence of a dangerous phenomenon of resonance.

3. The natural oscillations of toroidal steel shells of pipelines with a liquid flow for various values of the relative curvature $\frac{r}{R}$, thinness $\frac{h}{r}$ pipes and different speeds $U$

flowing fluid. Analysis of the results of the studies allowed us to draw the following conclusions:

- Flow rate $U$, varying in the range of real velocities of the 
liquid flowing in the pipeline (up to $20 \frac{\mathrm{m}}{\mathrm{s}}$ ), little effect on the frequencies of natural oscillations of the curvilinear sections of the steel pipeline over all the shell shapes studied ( $m, n=1,2,3$ at $n=1,2,3)$. Oscillation frequencies $\omega_{m n}$ decrease with an increase in the flow rate from 0 to $20 \frac{\mathrm{m}}{\mathrm{s}}$ not more than $7 \%$.

\section{References}

[1] Bozorov M. B., Safarov I. I., Shokin Yu. I. Numerical simulation of oscillations of dissipative homogeneous and inhomogeneous mechanical systems. Siberian Branch of the Russian Academy of Sciences, Novosibirsk, 1966-188 p.

[2] Safarov I. I., Akhmedov M., Umarov A. Own vibrations of toroidal shell with flowing liquid. Lambert Academic Publishing (Germany). 2017. 177p. hhtp:// dnb.d -nb.de. ISBN: 978-3-330-06423-2

[3] Vlasov V. Z. General theory of shells and its applications in engineering. - Moscow-Leningrad. Gostekhizdat Press., 1949$784 \mathrm{p}$.

[4] Volmir AS, Grach M. S. Fluctuations of a shell with a flowing fluid, Izvestiya USSR Academy of Sciences, Mechanics of Solid State, No. 6, 1973.-p. 162-166.

[5] Vol'mir AS, Shells in a stream of liquid and gas. Problems of aeroelasticity. - Moscow: Nauka, 1976.-416 p.

[6] Galiev Sh. U. Dynamics of the interaction of structural elements with the pressure wave in the liquidity. - Kiev: Nauka Dumka press. 1977172 p.

[7] Gladkikh P. A, Khachaturyan S. A. Vibration in pipelines and methods for their elimination. Moscow, Mashgiz press, 1969. $170 \mathrm{p}$.

[8] Gol'denveizer A. L. The theory of elastic thin shells. Moscow, Gostehizdat press. 1953, -544 p.

[9] Kayumov S. S., Safarov I. I. Propagation and diffraction of waves in dissipative - inhomogeneous cylindrical deformable mechanical systems. Tashkent, Publishing house: Science, 2004, 214 p.

[10] Safarov I. I., Nuriddinov B. Z., Shodiyev Z. O. Dynamic stress-Deformed condition layer cylindrical layer from the harmonic wave. World Wide Journal of Multidisciplinary Research and Development (WWJMRD). 25, 2017, 3(7) P.277-286 www.wwjmrd.com

[11] SNIP 2.05.06-85 *. Migratory pipelines.- M.: Gosstroy of Russia, 1997. 60 p.
[12] Safarov I. I., Teshayev M. K., Boltayev Z. I., Akhmedov M. Sh. Damping Properties of Vibrations of Three-Layer VIscoelastic Plate. International Journal of Theoretical and Applied Mathematics 2017; 3(6): 191-198 http://www.sciencepublishinggroup.com

[13] Safarov I. I., Teshaev M. X. Akhmedov M. Sh., Ruziyev T. R Application Of The Method Of Finite Element For Investigation Of The Dynamic Stress- deformed Condition Of Pipeline Sides When Exposed External Loods. // Case Studies Journal -Volume 6, Issue-5-May-2017. P.38-4514.

[14] Safarov I. I., Teshaev M. KH, Boltaev Z. I.. Mathematical modeling of wave process in a mechanical waveguide taking into account the internal friction. Germany. LAP. 2013. 243p.

[15] Safarov I. I, Akhmedov M. Sh., Boltaev. Z. I. Dissemination Sinusoidal Waves in of A Viscoelastic Strip. Global Journal of Science Frontier Research: F Mathematics \& Decision Sciences. 2015. Volume 15 Issue 1 (Ver.1.0). P.39-60.

[16] Safarov I. I, Akhmedov M. Sh., Boltaev. Z. I. Ducting in Extended Plates of Variable Thickness. Global Journal of Science Frontier Research: F Mathematics \& Decision Sciences. 2016. Volume 16 Issue 2 (Ver.1.0). P.33-66.

[17] Koltunov M. A.. Creep and relaxation. - M.: Higher School press, 1976.-276p.

[18] Safarov I. I., Teshaev M. KH., Boltaev Z. I. Distribution of linear waves in extended lamellar bodies. LAP, Lambert Academic Publishing (Germany). 2016. 315 p.

[19] Safarov I. I., Akhmedov M. Sh., Boltaev Z. I.. Proper waves in layered media. Lambert Academic Publishing (Germany). 2016. 192p.

[20] Safarov I. I, Boltaev Z. I., Akhmedov M. Sh. Properties of wave motion in a fluid-filled cylindrical shell/ LAP, Lambert Academic Publishing. 2016-105 p.

[21] Safarov I. I, Akhmedov M. Sh., Qilichov O. Dynamics of underground hiheline from the flowing fluid.. Lambert Academic Publishing (Germany). 2016. 345p.

[22] S. K. Godunov. On the numerical solution of boundary value problems for systems of linear ordinary differential equations. - Successes of Mathematical Sciences, 1061, T. 16, № 3, 171$174 \mathrm{p}$.

[23] Bolotin V. V. Oscillations and stability of an elastic cylindrical shell in a flow of a compressible fluid. -Inzh. sb., 1956, v. 24, p. 331

[24] Bolotin V. V. Dynamic stability of elastic systems. -Moscow, Gostekhizdat press, 1956.-600 p. 